\title{
Reanalysis of the 1992 South Pole Millimetre-Wavelength Atmospheric Opacity Data
}

\author{
Richard A. Chamberlin ${ }^{\mathrm{A}, \mathrm{B}}$ \\ A Caltech Submillimeter Observatory, Hilo, HI 96720, USA \\ B E-mail: cham@submm.caltech.edu
}

Received 2003 January 20, accepted 2004 March 11

\begin{abstract}
In 1992 an NRAO 225-GHz site survey heterodyne radiometer was placed at the Geographical South Pole. The instrument operated over an entire annual cycle and provided direct measurements of the millimetre-wave sky brightness temperature as a function of zenith angle. Interpreted in a single-slab 'skydip' radiation transfer model of the atmosphere, these sky brightness measurements provided a time series of the millimetre atmospheric opacity. Statistics derived from this opacity time series were important for making comparisons with other candidate millimetre and sub-millimetre wave astronomy sites. This paper reexamines the 1992 measurements and the original analysis. Details of the skydip fit model, radiometer gain error, instrument stability, and a mid-season replacement to a window in the instrument enclosure combined to cause a modest under-reporting of the atmospheric opacity in previous reports. Unchanged are earlier conclusions that dry air makes a significant contribution to the total opacity at $225 \mathrm{GHz}$.
\end{abstract}

Keywords: instrumentation: miscellaneous — methods: data analysis — site testing — atmospheric effects

\section{225-GHz Opacity at the South Pole}

In 1992, a National Radio Astronomy Observatory (NRAO) 225-GHz heterodyne site testing radiometer ( ' $\tau$ meter'; Liu 1987; McKinnon 1987) was placed at the South Pole. This instrument provided measurements of the millimetre sky opacity for an entire annual cycle (Chamberlin \& Bally 1994, 1995). One result of these measurements was the finding of a significant dry-air contribution to the millimetre-wave atmospheric opacity (Chamberlin \& Bally 1995). The existence and approximate magnitude of this dry-air component was confirmed by South Pole opacity measurements made near $273 \mathrm{GHz}$ (R. de Zafra, private communication 1994) and by later measurements with the Antarctic Submillimeter Telescope and Remote Observatory (AST/RO) instrument (Chamberlin 2001).

The finding of significant dry-air opacity in site surveys has recently called into question by Calisse (2004) who suggested that incorrect modelling of 'radome' opacity may lead to over-estimates of atmospheric opacity: “... unless radome transparency is correctly modelled, some previous site studies may have significantly underestimated the quality of the best submillimetre sites ...". 1 There is an implication that incorrect modelling of radome opacity may be responsible for the magnitude of the dry-air terms reported previously (e.g. Chamberlin \& Bally 1995; Chamberlin et al. 1997; Chamberlin 2001). Concerns about uncompensated window opacity expressed

${ }^{1}$ From Calisse (2002), published Calisse (2004). by Calisse and others (e.g. R. A. Chamberlin to J. Peterson, private communication 2001 October) may apply to opacity results reported at $857 \mathrm{GHz}$ (Peterson et al. 2003), but as will be shown in Section 4, Calisse's specific concerns do not apply to results previously reported from other South Pole opacity measurements.

\section{The 225-GHz Radiometer}

The hardware and data acquisition method used by the site test radiometer are well described in Liu (1987) and Chamberlin \& Bally (1994). Of interest is the effect of a mylar window installed in the instrument enclosure between the internal calibration load chopper system and the external, off-axis paraboloidal tipping mirror. As originally installed, this mylar window was very thin and is believed to have made only a modest contribution to the received signal, perhaps about $5 \mathrm{~K}$. The thickness of the original mylar membrane was not measured. However, we believe it was thin because it broke very easily and inspection of the signal from loads of known temperature (see Section 5) showed no evidence of a bias toward warmer radiometer signal temperatures. Measurements with a Caltech Submillimeter Observatory facility receiver (J. Kooi, private communication) show that $12-25 \mu \mathrm{m}$ thick mylar membranes in the $230-\mathrm{GHz}$ radio beam add about 5 to $10 \mathrm{~K}$ to the receiver noise temperature. The mylar opacity implied by that noise temperature increase is about 0.01 to 0.02 nepers. Here, nepers to refers to power attenuation (Kraus 1986). A low-opacity window would have 
had almost no effect on our original method of computing atmospheric opacity (Chamberlin \& Bally 1994), see Section 3 . The original, thin window was punctured by mechanical cleaning and replaced with a thicker and more emissive mylar film on Day 90 (1992 March 30). The effects of the this window replacement were only partly accounted for in the earlier analysis (Chamberlin \& Bally 1994).

\section{Skydip Fitting Models}

In Chamberlin \& Bally (1994) the skydip data-fitting routine was presented with calibrated data in the form $T_{\text {sky }}(A)$ versus $A$, where $T_{\text {sky }}(A)$ was the apparent sky temperature and $A$ was the airmass which depends zenith angle $Z A$, such that $A \approx 1 / \cos (Z A)$. $T_{\text {sky }}(A)$ included sky emissivity as well as emissivity from all other sources which terminated the radio beam beyond the calibration loads.

In Chamberlin \& Bally (1994) the skydip data $\left(T_{\text {sky }}(A)\right.$ versus $A$ ) were fitted to

$$
T_{\text {sky }}(A)=T_{0}+T_{\text {sur }}\left[1-\exp \left(-\tau^{\prime} A\right)\right]
$$

where $T_{0}$ was the combined contribution to excess signal noise from mylar window emission, ground spillover, etc. (i.e. $T_{0}$ was a static contribution to $T_{\text {sky }}(A)$ independent of $A), \tau^{\prime}$ was the apparent sky opacity, and $T_{\text {sur }}$ was the atmospheric temperature measured at the instrument enclosure. Figure 1 is a reproduction (Chamberlin \& Bally 1994) of an example of a typical 1992 skydip measurement and fit to Equation (1). The second term on the right hand side of Equation (1) is merely an application of phenomenological radiation transfer modelling (Rohlfs 1986 p. 7) to the atmosphere, and it is the so-called single-slab skydip model.

A more appropriate fit equation would have been

$$
T_{\text {sky }}(A)=T_{0}+\exp \left(-\tau_{\text {window }}\right) T_{\text {atm }}[1-\exp (-\tau A)]
$$

which is basically the same as Equation (1) except that the portion of the signal which passes through a thin mylar window in the instrument enclosure is attenuated by the

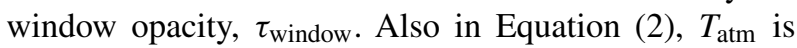
used instead of $T_{\text {sur }} . T_{\text {atm }}$ is the properly weighted average temperature computed from daily radiosonde data - see the Appendix. At middle latitude sites, generally $T_{\mathrm{atm}}<$ $T_{\text {sur }}$ due to the normal adiabatic lapse rate of the atmosphere. In contrast, at the South Pole, typically $T_{\text {atm }}>T_{\text {sur }}$ because of the deep inversion layer which forms most of the year and particularly in winter (Schwerdtfeger 1984; Chamberlin 2001; Chamberlin et al. 2002). In Equation (2) opacity $\tau$ instead of $\tau^{\prime}$ is obtained.

Skydip models like Equation (2) are widely used in radio astronomy often with many extra terms added to account for instrumental effects, such as increases in system noise temperature due to emissive membranes (Meeks \& Ruze 1971) and ground spillover, and antenna gain losses due to radome attenuation (Meeks \& Ruze 1971), beam coupling efficiency, and so forth (Ulich \&

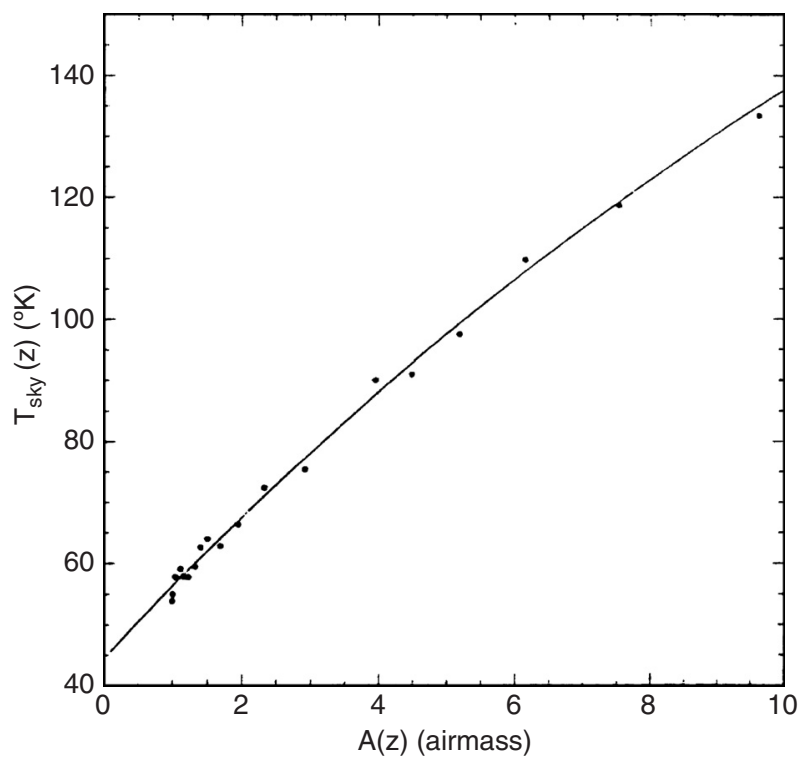

Fig. 2. This graph shows the results of a typical zenith-to-horizon sky-brightness measurement scan. The solid points are radiometric measurements of the sky-brightness temperature. The line is a fit of the Eq. (2) to these data. According to this fit, $:=0.056$ air mass $^{-1}$, and $T_{0}=44.4 \mathrm{~K}$, with $T_{\text {at }} \equiv 217.5 \mathrm{~K}$. The standard deviation of the averaged measurements for each point was less than $0.5 \mathrm{~K}$; therefore, no error bars are shown.

Figure 1 225-GHz skydip figure, reproduced from Chamberlin \& Bally (1994).

Haas 1976). For just one example of a more elaborate form of Equation (2), see Equation (II-4) in Lane (1982). ${ }^{2}$

Since $\exp \left(-\tau_{\text {window }}\right)$ is an instrumental constant for convenience, we can define $\eta \equiv \exp \left(-\tau_{\text {window }}\right)$ and rewrite Equation (2) as

$$
T_{\text {sky }}(A)=T_{0}+\eta T_{\text {atm }}[1-\exp (-\tau A)]
$$

Under low 225-GHz opacity skies, such as at the South Pole, $\tau \ll 1$, and at low airmass Equations (1) \& (3) can

${ }^{2}$ Equation (II-4) from Lane (1982) was developed for use in the skydip/calibration program written for the Five College Radio Astronomy Observatory (FCRAO) (Lane 1978). For convenience, we repeat it here:

$$
\begin{aligned}
T_{\mathrm{obs}}(A)= & T_{\mathrm{rx}}+T_{\mathrm{rad}}\left[1-\exp \left(-\tau_{\mathrm{rad}}\right)\right]+\left(1-\eta_{l}\right) T_{\mathrm{amb}}\left[\exp \left(-\tau_{\mathrm{rad}}\right)\right] \\
& +\eta_{l} T_{\mathrm{atm}}\left[\exp \left(-\tau_{\mathrm{rad}}\right)\right]\left[1-\exp \left(-\tau_{\mathrm{o}} A\right)\right]
\end{aligned}
$$

$T_{\mathrm{obs}}(A)$ corresponds to the total noise power received at airmass $A$. As we can see from this Equation, the total noise power has contributions from receiver noise $T_{\mathrm{rx}}$, radome emission $T_{\mathrm{rad}}\left[1-\exp \left(-\tau_{\mathrm{rad}}\right)\right]$, ground spillover from outside the radome $\left(1-\eta_{l}\right) T_{\mathrm{amb}}\left[\exp \left(-\tau_{\mathrm{rad}}\right)\right]$, and from the directed power on the sky $\eta_{l} T_{\mathrm{atm}}\left[\exp \left(-\tau_{\mathrm{rad}}\right)\right]\left[1-\exp \left(-\tau_{\mathrm{o}} A\right)\right]$. In this context, the term $\eta_{l}$ was called the 'antenna loss efficiency' and represents the fraction of power from outside the radome which was received in the directed radio beam, that is $\eta_{l}$ is the fraction of power from outside the radome which changes with airmass $A$. In earlier literature (Davis \& Vanden Bout 1973), $\eta_{l}$ "refer(s) to loss mechanisms, i.e., spillover, blockage, and Ohmic losses." Accordingly, it would be natural to include radome loss in $\eta_{l}$, but in Lane (1982) radome contribution to efficiency loss was explicitly quantified in the skydip model probably because it was relatively easy to do so. $T_{\mathrm{amb}}$ is the ambient air temperature, $T_{\mathrm{atm}}$ the properly weighted atmospheric temperature, $\tau_{\mathrm{o}}$ the zenith opacity, $T_{\text {rad }}$ the radome temperature, and $\tau_{\text {rad }}$ the radome opacity. 
be well approximated by the linear forms

$$
T_{\text {sky }}(A)=T_{0}+T_{\text {sur }} \tau^{\prime} A
$$

or

$$
T_{\text {sky }}(A)=T_{0}+\eta T_{\text {atm }} \tau A
$$

where $\tau^{\prime}$ is the apparent sky opacity when window opacity is neglected and $T_{\text {sur }}$ is used, and $\tau$ is a more accurate sky opacity which would have been found if Equation (3) was used to fit the skydip data. We can see from Equation (5), that for the very low opacities typical of winter, the initial slope of the skydip, $T_{\text {sky }}(A)$ versus $A$, is the product $\eta T_{\text {atm }} \tau$.

It is obvious from comparing Equations (4) \& (5) that

$$
\tau=\frac{T_{\text {sur }}}{T_{\text {atm }}} \frac{\tau^{\prime}}{\eta}
$$

If $T_{0}$ is dominated by window emission as apparently was the case after Day 90 when the window was replaced, again applying the phenomenological radiation transfer model (Rohlfs 1986 p. 7) we get

$$
T_{0}=T_{\text {window }}(1-\eta)
$$

or

$$
\eta=1-T_{0} / T_{\text {window }}
$$

where $T_{\text {window }}$ was the physical temperature of the window. From inspection of the zero intercept in data like Figure 1, previously we found that $T_{0} \simeq 45 \mathrm{~K}$ subsequent to the window replacement on Day 90 (Chamberlin \& Bally 1994).

The instrument enclosure temperature was maintained at $10^{\circ} \mathrm{C}$ by an external heating system (Chamberlin \& Bally 1994). So $T_{\text {window }}$ was some intermediate temperature between the enclosure temperature and $T_{\text {sur }}$. For our analysis here we assumed $T_{\text {window }}=250 \mathrm{~K}$. This assumed value of $T_{\text {window }}$ was used because it is intermediate between the enclosure temperature and the average wintertime surface temperature - in 1992, Days 100 to 300, $\left\langle T_{\text {sur }}\right\rangle=216.4 \pm 9.3 \mathrm{~K}$. If the assumed value of $T_{\text {window }}$ is used, from Equation ( 8 ) we obtain $\eta \approx 1-45 / 250=0.82$, suggesting that the previously reported opacities (Chamberlin \& Bally 1994) may be about $18 \%$ too low from neglecting window opacity in Equation (1).

Somewhat mitigating possible earlier underreporting of the opacities is that fact that $T_{\text {sur }}$ is often significantly lower than $T_{\text {atm }}$ due to the deep inversion layer which typically forms at the surface. For example, during the lowest opacity weather, it is often the case that $T_{\text {sur }} \approx 205 \mathrm{~K}$ and $T_{\text {atm }} \approx 230 \mathrm{~K}$. Thus from Equation (6)

$$
\tau=\frac{0.89}{0.82} \tau^{\prime}=\frac{\tau^{\prime}}{0.92}
$$

implying that, during the best weather periods, the earlier underreporting of opacity (Chamberlin \& Bally 1994) was on the order of $10 \%$.

\section{Comparison to Analysis in Calisse (2004)}

In Calisse (2004) it is suggested that window-induced instrumental artifacts may be responsible for excess 'dryair' opacity at very good submillimetre sites where water vapor opacity is very low. The abstract and conclusion in the Calisse paper suggest that results from site surveys other than those accomplished with the CMU/NRAO $350-\mu \mathrm{m} \tau$-meter may be affected (Peterson et al. 2003). However, other than the CMU/NRAO instrument, we do not know of any other South Pole site survey results that are affected by the specific analysis in Calisse (2004).

For example, in the AST/RO instrument (Stark 2001; Chamberlin 2001) no windows were used in the beam path from the calibration system to the sky, thus the window opacity effect described in Calisse does not apply.

In a South Pole atmospheric Fourier transform spectrometer (Chamberlin et al. 2002), a removable window was usually in the beam path between the cold load chopper and the external tipping mirror but the effects of window emission and absorption were included in the data analysis.

Regarding the topic of this paper, 1992 opacity results from a NRAO 225-GHz $\tau$-meter, a window with a nonnegligible absorption was used during a part of 1992 , but Calisse's analysis (Calisse 2004) does not apply here either. Calisse's Equation (1) assumes a modelling error of the form

$$
T=T_{\text {atm }}\left[1-\exp \left(-\tau^{\prime \prime} A\right)\right]-T_{\text {ref }}
$$

or, equivalently

$$
T_{\mathrm{sky}}(A)=T_{\mathrm{atm}}\left[1-\exp \left(-\tau^{\prime \prime} A\right)\right]
$$

with $T_{\text {sky }} \equiv T+T_{\text {ref }}$. In Calisse (2004) $T_{\text {ref }}$ is the blackbody temperature of a synchronously detected calibration load. Equation (11) is not the same as Equation (1). Equation (11) neglects the emissive contribution from the instrument window which was not an error made in the previous analysis of the 1992 225-GHz skydip data set. That is to say, the fit parameter $T_{0}$ in Equation (1) includes window emissivity. If the radiometer performance is ideal in every other respect, then $T_{0}$ is related to the window opacity by Equation (7). Since $T_{0}$ includes window emissivity, it is similar to using the second term on the right hand side of Equation (5) of Calisse (2004) in his preferred form of the skydip model.

To illustrate the point that Equation (1) does not make the modelling error discussed by Calisse, consider Figure 2. The solid line is the fit of Equation (1) to the skydip data and it gives $T_{0}=44.4 \mathrm{~K}$ and $\tau^{\prime}=0.056 . T_{\text {sur }}$ was constrained to be the surface temperature $217.5 \mathrm{~K}$. In contrast, the dashed line shows the result of the least-squared best fit to Equation (11) and it gives $\tau^{\prime \prime}=0.145$. $T_{\text {atm }}$ is constrained to be the surface temperature $217.5 \mathrm{~K}$ but clearly no reasonable choice of $T_{\text {atm }}$ could make Equation (11) appropriate to use on this skydip data. If the same skydip data is fit to Equation (3) (dotted line), we find that $T_{0}=43.6 \mathrm{~K}$ and $\tau=0.067$ with $\eta$ constrained to be 0.82 


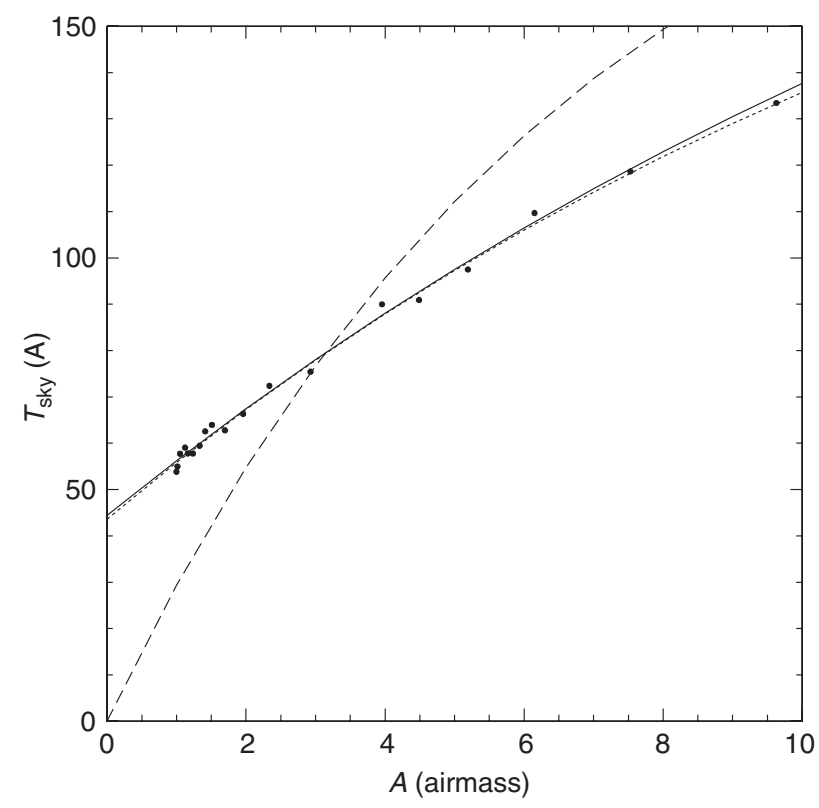

Figure 2 Different model fits to 225-GHz skydip data from Figure 1. The solid line is a fit to Equation (1), the dotted line is a fit to Equation (3), and the dashed line is a fit to Equation (11).

and $T_{\text {atm }}$ constrained to be $230.0 \mathrm{~K}$, which is near the average value determined from radiosonde. As expected from Section 3, the error in the opacity determination from using model Equation (1) instead of Equation (3) was moderate and $\tau^{\prime}<\tau$.

\section{Other NRAO 225-GHz $\tau$-Meter Systematic Errors}

In the previous Sections we saw that uncompensated transmission loss through a non-ideal window put in the signal beam path after Day 90 had the likely effect of moderately reducing the $225-\mathrm{GHz}$ sky opacities reported in Chamberlin \& Bally (1994) and Chamberlin et al. (1997).

We also explained that using a properly weighted atmospheric temperature, rather than just the surface temperature, made the skydip model we use more realistic.

Besides uncompensated transmission loss through a window in the instrument enclosure, other systematic errors can result from gain error, gain drift, snow on the optics, and perhaps some other sources not yet identified.

\subsection{The Chopper Calibration System and Validation with $\mathrm{LN}_{2}$ Test Loads}

As described previously (Liu 1987; Chamberlin \& Bally 1994), the NRAO $225-\mathrm{GHz} \tau$-meter has a four-bladed chopper system which switches the input of the radiometer between two reference loads and the sky signal from the external mirror. The gain of the radiometer system was established by synchronously detecting its output power with the input switching between thermally controlled 45 and $65^{\circ} \mathrm{C}$ 'reference' and 'hot' loads. Actual sky brightness temperatures can be lower than $-263^{\circ} \mathrm{C}$ so the gain established by comparing two warm reference loads had to be extrapolated to much colder temperatures.
To check that whole system was giving reasonable values, during the first few months of operation the $\tau$-meter was periodically monitored with a liquid nitrogen $\left(\mathrm{LN}_{2}\right)$ load until the $\mathrm{LN}_{2}$ at the South Pole station ran out on Day 73. The average of 97 separate measurements combined gave $T_{\mathrm{LN}_{2}}=74.8 \pm 10.7 \mathrm{~K}$. The large uncertainty was dominated by long-term gain drifts between monitoring periods, rather than variation between consecutive measurements. These measurements of the liquid nitrogen load were accomplished with the radiometer looking through its original, thin mylar window (see Section 2).

The latent heat of evaporation and the boiling temperature of $\mathrm{LN}_{2}$ (at sea-level pressures) are established so we used the Clausius-Clapeyron equation to extrapolate the pressure versus temperature liquid/gas coexistence curve to a typical South Pole surface pressure of 690 mbar. Doing so, we found that $T_{\mathrm{LN}_{2}}=74.1 \mathrm{~K}$, very close to the temperature the radiometer measured. However, the measurements were made through the original, thin mylar window, which was expected to add about 2 to $4 \mathrm{~K}$ to the observed signal (see Section 2 ) ${ }^{3}$ thus indicating a slight radiometer gain error of 1 to $2 \%$ and a tendency to measure a lower than expected signal temperature.

\subsection{Other Measurements in the Data Stream}

Besides doing skydips, the 1992 data stream from the radiometer was programmed to include periods when it monitored an outside ambient surface temperature load at $Z A=-45^{\circ}$, and the cold zenith sky at $Z A=0^{\circ}$. Also reported in the data stream was the radiometer gain. These data, not reported previously, are shown in Figure 3. $G_{\mathrm{Z}}$ (Figure 3, Panel A) was the radiometer gain with the external tipping mirror on the cold zenith sky, $Z A=0^{\circ}$. $G_{\mathrm{A}}$ (Panel B) was the radiometer gain with external tipping mirror directed onto the ambient load, $Z A=-45^{\circ}$. $T_{\mathrm{Z}}$ (Panel C) was the measured zenith sky temperature. $T_{0}$ is the excess temperature found from fitting Equation (1) to skydip data. $T_{\mathrm{amb}}-T_{\mathrm{AD} 590}$ was the difference between radiometer's measurement of the external load temperature and the actual load temperature, assumed equal to the value detected at an AD590 sensor just below the external mirror. The units of radiometer gain (Panels $\mathrm{A} \& \mathrm{~B}$ ) are $\mathrm{mV} \mathrm{K}^{-1}$. The units of temperature (Panels $\mathrm{C}$, $\mathrm{D}, \& \mathrm{E})$ are K. For use in Equation (1), and for other comparisons made here, $T_{\mathrm{AD} 590}$ was assumed equal to the surface temperature $T_{\text {sur }}$.

In Figure 3, quantities are boxcar-averaged with a few adjacent points to reduce noise. At Day 90, a sudden increase in $T_{0}, T_{\mathrm{Z}}$, and $T_{\mathrm{amb}}$ was evident and it was due to the installation of the replacement window which was more opaque.

\footnotetext{
${ }^{3}$ Whether or not the window adds much to the observed temperature depends on the difference between window and source temperature. If we define $T_{\text {excess }}$ as the difference between the observed temperature and the source temperature, $T_{\text {source }}$, then it is easy to show that $T_{\text {excess }}=\left(T_{\text {window }}-T_{\text {source }}\right)\left[1-\exp \left(-\tau_{\text {window }}\right)\right]$, or $T_{\text {excess }}=\left(T_{\text {window }}-T_{\text {source }}\right) \tau_{\text {window }}$ when $\tau_{\text {window }} \ll 1$.
} 

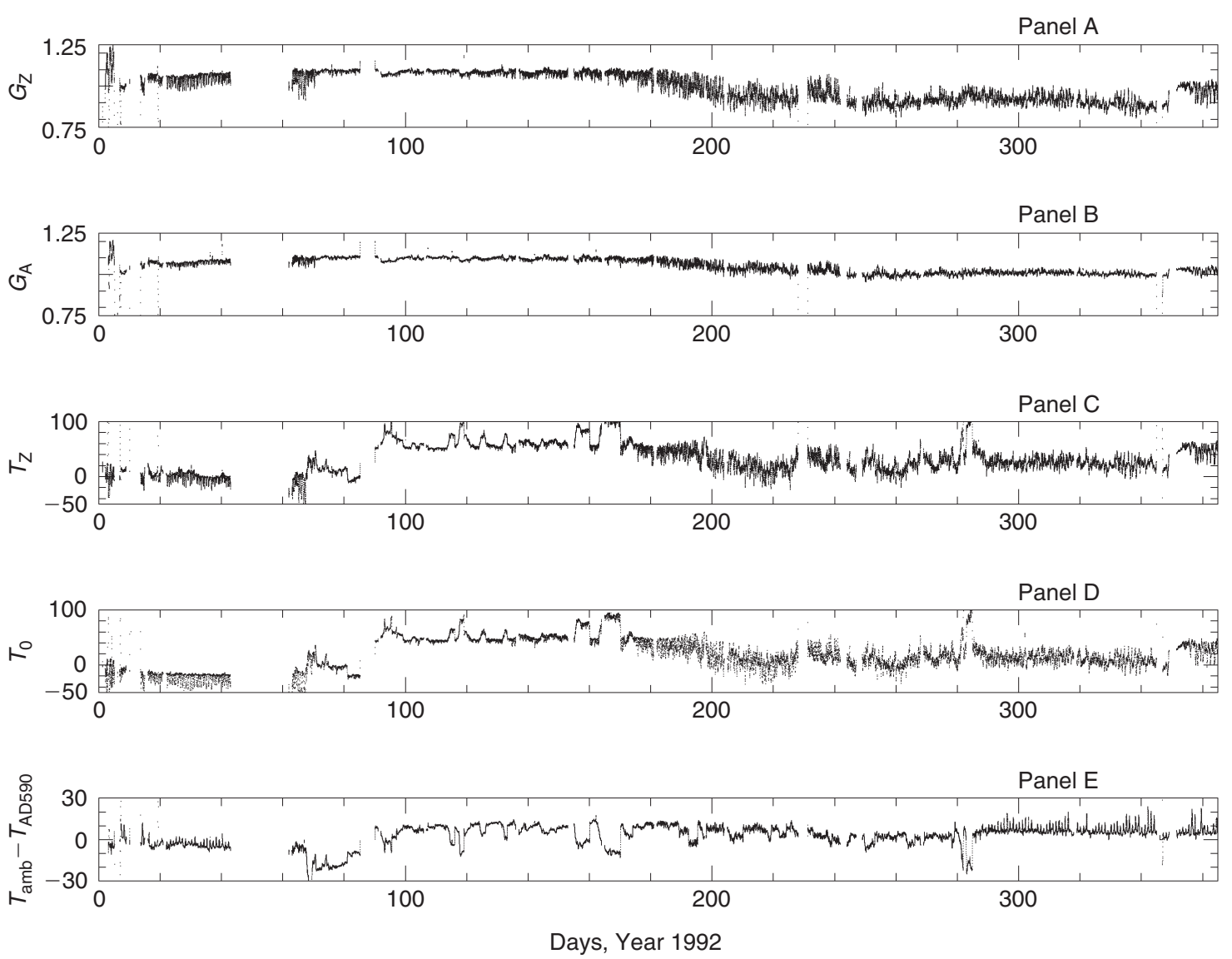

Figure 3 A time series of some useful diagnostic indicators. See Section 5 definitions and discussion.

\subsection{Chopper Phase Drift after Day 180}

After about Day 180 there is evidence of change in the radiometer gain due to loss of phase in the chopper calibration system. We observe that before Day $180, G_{\mathrm{Z}}$ and $G_{\mathrm{A}}$ were in good agreement, but deviate after that. That is, the indicated radiometer gain decreased and depended on the signal temperature(!). $G_{\mathrm{Z}}$ was obtained with the external mirror aimed at the cold zenith sky, and $G_{\mathrm{A}}$ was obtained with the external mirror aimed at the much warmer ambient surface temperature load.

In an ideal chopper calibration system, loss of phase would lead to a decrease in gain, but the gain is not expected to depend on the external signal temperature. If some external signal enters into the chopper synchronous detection system due to poor chopper wheel phasing, its contribution should cancel if its contribution is symmetric in both the hot load and cold load chopper phases.

Lui (1987) observed and noted the load dependent gain when the chopper is out of phase, but he did not provide an explanation. We believe the explanation is this: the chopper wheel is four bladed and the blades are tilted with respect to the beam axis. During the transitioning part of the chopper cycle the beam is partly on the load, partly on the exit window, and possibly diffracted onto other surfaces in the instrument enclosure. When transitioning from the reference load to the sky, and transitioning from the hot load to sky, the optical situation is not symmetric.
The chopper blades are tilted with respect to the beam in different directions in these two transition cases so as to direct the radiometer to either the reference load which is at about $90^{\circ}$ to the interrupted beam direction, or direct it to the hot load which is opposite at about $-90^{\circ}$. That is, during the transition period, the size of the illuminated spot on the exit window to the sky depends not only chopper phase but also whether the transition is from the reference load to the sky, or hot load to the sky. During the period of proper operation, Days 4-180, this transition part of the chopper phase was blanked out and not accepted by the synchronous detection system. Thus, skydip data from Days 4-180 may have more reliable calibration than after Day 180 (see Sections 6.4 and 6.5).

\subsection{Snow Accumulation on the Optics}

There is evidence of short periods of snow accumulation on the window and external mirror leading to increased $T_{0}$ and increased zenith sky temperature $T_{Z}$. This snow accumulation also scattered the beam which was manifested by decrease in $T_{\mathrm{amb}}-T_{\mathrm{AD} 590} . T_{\mathrm{amb}}$ was the radiometer temperature of the ambient load suspended at $Z A=-45^{\circ}$ with the sky behind it rather than the ground as noted above in Section 5.2. Beam broadening due to snow scatter on the external optics thus led to some of the beam being spilled over onto the cold sky behind the load. In this way, snow accumulation on the external 

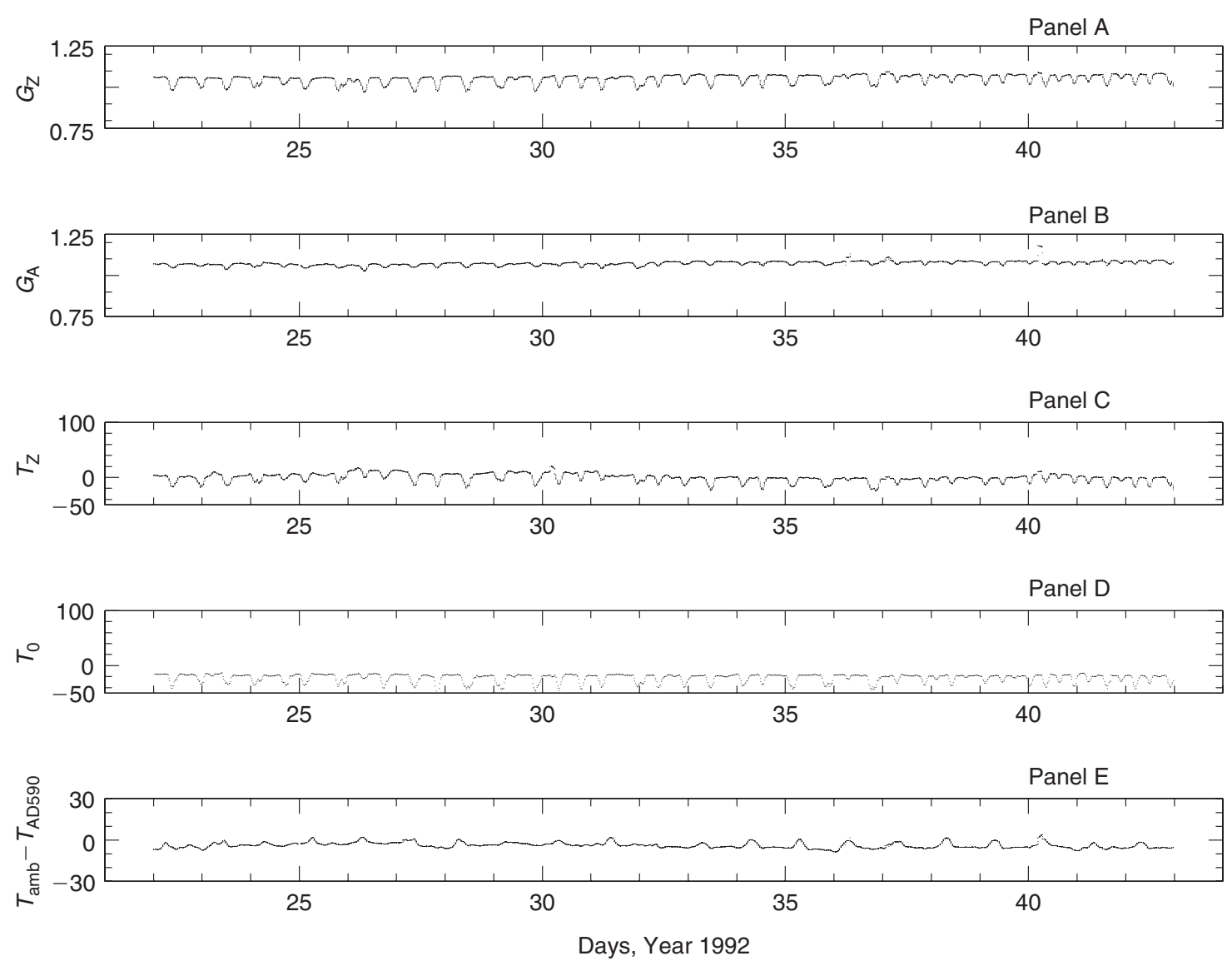

Figure 4 A time series of some useful diagnostic indicators: an expansion of the previous figure on Days 22-43. See Section 5 definitions and discussion.

optics was indicated by increase of $T_{0}$ and $T_{\mathrm{Z}}$ correlated with decrease in $T_{\mathrm{amb}}-T_{\mathrm{AD} 590}$ (e.g. in Figure 3 see Days 93, 95, 114-116, 117-119, 124-126, 132-133, 155-160, and 164-170). The snow accumulation was periodically cleaned off the external optics by the technician monitoring the instrument.

\subsection{More Indications of a Small Systematic Gain Error}

As indicated above in Section 5.1, even before Day 180, there may have been a systematic radiometer gain error. To better quantify that possible error, we note that in Figure 3, there is a fairly stable period between Days 22 and 43 . In Figure 4 we show that period in more detail. As previously, adjacent points are smoothed into two-hour running averages to reduce noise and make trends more visible. From the data in Figure $4\left\langle G_{\mathrm{Z}}\right\rangle=1.05 \pm 0.03 \mathrm{mV} \mathrm{K}^{-1}$ and $\left\langle G_{\mathrm{A}}\right\rangle=1.07 \pm 0.03 \mathrm{mV} \mathrm{K}^{-1}$. As it should be, if the chopper calibration system was properly phased during this period, $\left\langle G_{\mathrm{Z}}\right\rangle \approx\left\langle G_{\mathrm{A}}\right\rangle$.

From the zenith sky temperature from Figure 4 we obtain $\left\langle T_{Z}\right\rangle=-0.6 \pm 9.4 \mathrm{~K}$ whereas we would expect the number to be about 12 to $17 \mathrm{~K}$ under a good sky when emission from the thin mylar window is included. About a $5 \%$ radiometer gain error is indicated.
If the radiometer gain was ideal, then $T_{0}$, the excess temperature from Equation (1), should be dominated by window opacity. From our earlier estimate of $\tau_{\text {window }}$ for a very thin mylar membrane (Section 2 ) we would expect $T_{0} \sim 3-5 \mathrm{~K}$. However, $\left\langle T_{0}\right\rangle=-22.3 \pm 9.4 \mathrm{~K}$. If the difference between the expected and observed $T_{0}$ is due to gain error, then about a $9 \%$ gain error is indicated.

Finally, we can obtain an estimate of the gain error from comparing $T_{\mathrm{amb}}$ and $T_{\mathrm{AD} 590} .\left\langle T_{\mathrm{amb}}-T_{\mathrm{AD} 590}\right\rangle=-3.9 \pm$ $2.5 \mathrm{~K}$. In this case, since $T_{\text {window }} \sim T_{\mathrm{amb}}$, the thin mylar window made a relatively minor difference, only increasing $T_{\mathrm{amb}}$ by an estimated 0.2 to $0.4 \mathrm{~K}$. $\left\langle\left(T_{\text {ref }}-T_{\mathrm{amb}}\right) /\right.$ $\left.\left(T_{\text {ref }}-T_{\mathrm{AD} 590}\right)\right\rangle=1.05 \pm 0.03$, indicating a gain error of about $5 \%$. $T_{\text {ref }}$ is the fixed temperature of the $45^{\circ} \mathrm{C}$ reference calibration load.

As with $\mathrm{LN}_{2}$, the time series from $T_{\mathrm{Z}}, T_{0}$, and from $T_{\text {amb }}$ indicate a slight radiometer gain error and with a tendency for the radiometer to measure lower than expected temperatures.

\subsection{Periodicity in Data}

Inspection of the time series displayed in Figures 3 and 4 show a periodic structure which invites more analysis. For example, in Figure 3, Panel E, there is clearly periodic structure in the difference $T_{\mathrm{amb}}-T_{\mathrm{AD} 590}$ between Days 22 and 43 and after about Day 285. The expanded time scale 
in Figure 4 is useful for seeing the periodicity in more detail. Fourier analysis on the time series of $T_{\mathrm{amb}}-T_{\mathrm{AD} 590}$ between Days 22 and 43 shows strong peaks in the power spectrum at exactly 1,2 , and $3 \mathrm{dy}^{-1}$ corresponding to the diurnal cycle and its harmonics. The diurnal characteristic $T_{\mathrm{amb}}-T_{\mathrm{AD} 590}$ occurred during a period when the sun was up and so can likely be attributed to differential heating of the radiometer load and the AD590 temperature transducer.

In the expanded time scale of Figure 4, Panel A, we can also clearly see periodic structure $G_{\mathrm{Z}}$ and corresponding structure in $G_{\mathrm{A}}$ (Panel B) but with lower amplitude. This correspondence in periodicity, but differing amplitude between $G_{Z}$ and $G_{\mathrm{A}}$ may suggest periods of poor chopper phasing, but we do not know how this effect could come about periodically, and then recover on its own. Fourier analysis on the time series of $G_{Z}$ shows a dominant peak at $1.82 \mathrm{dy}^{-1}$, that is, a periodicity of 13.2 h. This periodicity is not conveniently linked to the diurnal cycle and we do not know what caused it. $G_{Z}$ was used to calibrate the sky signal so it not surprising that corresponding structure appears in $T_{\mathrm{Z}}$, Panel $\mathrm{C}$. However, it also appears in the derived quantity $T_{0}$ suggesting it may be a component introduced into the $\tau$ time series.

Fourier analysis of the $\tau$ time series derived using Equation (1) showed strong peaks in the power spectrum at 1.0, 1.8 , and $3.0 \mathrm{dy}^{-1}$ as well as other structure. It seems likely the peak at $1.8 \mathrm{dy}^{-1}$ was due gain variations in the instrument itself, rather than actual variations in the atmosphere. The peaks at 1.0 and $3.0 \mathrm{dy}^{-1}$ which are not evident in the $G_{\mathrm{Z}}$ power spectrum, may have been due to diurnal variation in the atmosphere or surface temperature.

\section{Reanalysis}

In Sections 3 and 4 we have seen that skydip data reduction may have been slightly biased toward lower values by the implicit assumption in Equation (1), that $\eta \equiv 1.0$, and slightly biased toward higher values by use of $T_{\text {sur }}$ instead of $T_{\text {atm }}$ in Equation (1). We estimated the net effect of using Equation (1) instead of Equation (2) was to bias the reported opacity about $10 \%$ toward lower values in the best weather.

In addition, in Section 5.3 we used previously unreported indications from the data stream to show that the data may be corrupted after about Day 180 due to loss of phase in the chopper calibration system. Prior to Day 43, during a stable period suitable for establishing instrument performance, we also inferred from $T_{\mathrm{Z}}, T_{\mathrm{amb}}$, and $T_{0}$ that the radiometer gain was somewhat in error, approximately $6 \%$, and biased toward measuring lower than true values for $T_{\mathrm{sky}}$.

\subsection{Correction of Gain Error}

If before Day 180 the radiometer gain error was approximately constant at $6 \%$, then we could apply a simple, constant gain correction and reanalyse the data. We did so and found the new results that between Days $22-43,\left\langle T_{\mathrm{Z}}\right\rangle=18.5 \pm 7.0 \mathrm{~K},\left\langle T_{0}\right\rangle=-1.18 \pm 6.9 \mathrm{~K}$, and $\left\langle T_{\mathrm{amb}}-T_{\mathrm{AD} 590}\right\rangle=0.8 \pm 1.7 \mathrm{~K}$. The constant $6 \%$ correction also gave $\left\langle G_{\mathrm{Z}}\right\rangle=1.12 \pm 0.03 \mathrm{mV} \mathrm{K}^{-1}$, and $\left\langle G_{\mathrm{A}}\right\rangle=$ $1.14 \pm 0.02 \mathrm{mV} \mathrm{K}^{-1}$. These corrected results for $T_{\mathrm{Z}}, T_{0}$, and $T_{\mathrm{amb}}$ are all more physically realistic.

With the gain correction applied, after the window replacement on Day 90 and before Day 180 we found $\left\langle T_{0}\right\rangle=69.2 \pm 13.1 \mathrm{~K}$, but this average was biased toward a higher value because it includes days when snow was collected on the enclosure window.

\subsection{Estimate of the Replacement Window Opacity}

Between Days 22-43, periods of bad calibration biased $\left\langle T_{0}\right\rangle$ toward a lower value (see Figure 4, Panel D). To obtain an estimate of $\left\langle T_{0}\right\rangle$ in the absence of bad calibration periods we applied the expected, fixed $6 \%$ gain correction and then examined the resulting distribution of $\left\langle T_{0}\right\rangle$ values (see Figure 5, Panel A). We found that the distribution had a sharp peak at $T_{0} \sim 2.8 \mathrm{~K}$, a full-width-half-maximum (FWHM) of $3.8 \mathrm{~K}$, and a long tail toward negative values. Thus, a fixed gain correction of $6 \%$ gave a result for $T_{0}$ which was in the range of the expected value: 2.5 to $5 \mathrm{~K}$.

Since the radiometer gain was relatively constant through to Day 180 (see Figure 3, Panels A \& B), we assume in the rest of this reanalysis that a gain correction of $6 \%$ applies.

With the radiometer gain correction applied we studied the distribution of $T_{0}$ after Day 90 when the replacement window was put in (see Figure 5, Panel B). This distribution was sharply peaked at $\sim 63.0 \mathrm{~K}$ with a FWHM of $7.2 \mathrm{~K}$ and a long tail toward higher values caused by periods of snow accumulation on the window. Using $T_{0}=63.0 \mathrm{~K}$ in Equation (8) we found that $\eta=0.748$, implying the opacity of the replacement window was $\tau_{\text {window }}=0.290$.

\subsection{Reanalysis Including Compensation for Major Known Errors}

The original 1992 opacity data were reanalysed with the following changes compared to Chamberlin \& Bally (1994, 1995):

1. A fixed $6 \%$ radiometer gain correction factor was applied to all data.

2. Before Day 90, we assumed $\tau_{\text {window }}=0.01$, and after window replacement on Day 90 we assumed $\tau_{\text {window }}=0.290$.

3. The skydip data, $T_{\text {sky }}$ versus A, were fit to Equation (2) instead of Equation (1).

\subsection{Comparison to Previously Reported Results}

The cumulative opacity statistics for the first half of 1992 (Chamberlin \& Bally 1994) were derived from skydip data modelled by Equation (1). The beginning of the lowopacity period which are most concerned with here started on about Day 70. The cumulative statistics from Chamberlin \& Bally (1994), along with the current reanalysis 

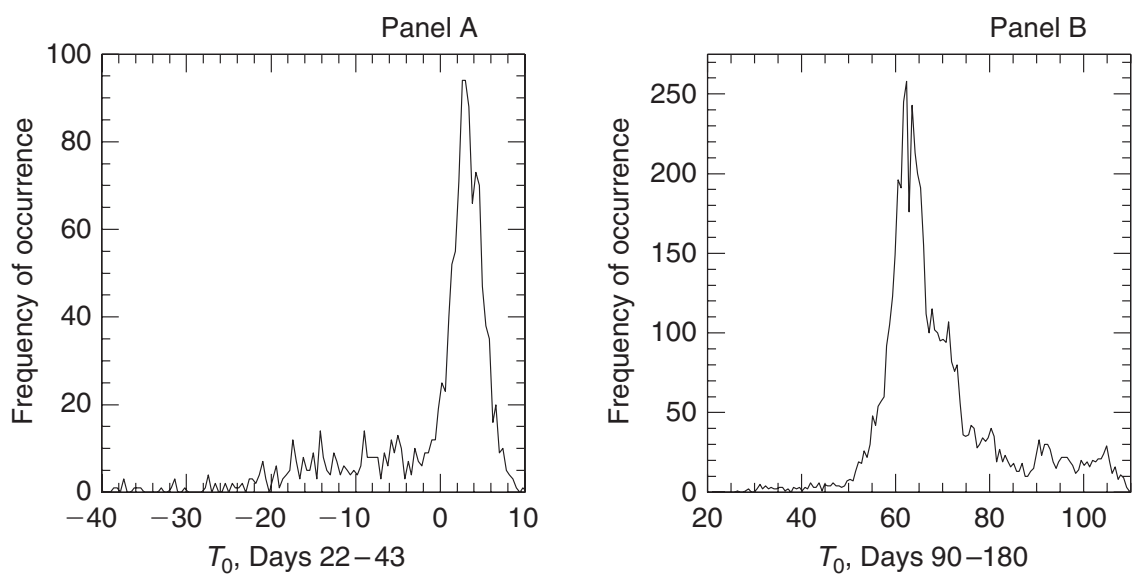

Figure 5 The distribution of $T_{0}$ values for Days 22-43 and Days 90-180. $T_{0}$ is in units of $\mathrm{K}$ and is a free parameter derived from the fit of Equation (1) or (2) to skydip data: $T_{\text {sky }}$ versus $A$. For data in this figure, prior to fitting to determine $T_{0}$, a $6 \%$ gain correction was applied to the radiometer gain, see Section 6.1. If $T_{0}$ was dominated by window emission, then $\eta$, the radiometer loss efficiency due to window opacity, can be determined from Equation (8). The amplitude of the plots, 'frequency of occurrence', is related to the total number of points in the sample and the resolution in $T_{0}$. These plot parameters are different in Panels A and B.

Table 1. Cumulative distribution for Days 70-180 in 1992

\begin{tabular}{llll}
\hline Percentile & \multicolumn{2}{c}{$\tau$} & Change \\
\cline { 2 - 3 } & $\begin{array}{l}\text { Chamberlin \& } \\
\text { Bally (1994) }\end{array}$ & This reanalysis & \\
\hline $25 \%$ & 0.039 & 0.045 & $+15 \%$ \\
$50 \%$ & 0.046 & 0.052 & $+13 \%$ \\
$75 \%$ & 0.055 & 0.064 & $+16 \%$ \\
$99.5 \%$ & 0.090 & 0.105 & $+16 \%$ \\
\hline
\end{tabular}

Table 2. Cumulative distribution for Days 100-321 in 1992

\begin{tabular}{llll}
\hline Percentile & \multicolumn{2}{c}{$\tau$} & Change \\
\cline { 2 - 3 } & $\begin{array}{l}\text { Chamberlin } \\
\text { et al. (1997) }\end{array}$ & This reanalysis & \\
\hline $25 \%$ & 0.040 & 0.049 & $+23 \%$ \\
$50 \%$ & 0.048 & 0.059 & $+23 \%$ \\
$75 \%$ & 0.057 & 0.072 & $+26 \%$ \\
$99 \%$ & 0.099 & 0.13 & $+31 \%$ \\
\hline
\end{tabular}

using the improved skydip model of Equation (2), are presented in Table 1. As indicated in the table, the reanalysis resulted in about a $15 \%$ increase in the opacity values at the quartiles.

In Chamberlin et al. (1997), Table 1, we reported the $225-\mathrm{GHz}$ opacity statistics for the entire 1992 winter/ spring period, Days 100-321. Those cumulative statistics along the current reanalysis for the same period using Equation (2) are shown in Table 2. Comparison to Table 1 shows increased opacities in all percentile categories when data from the second half of the year is included in the statistics. This result is unexpected because the second half of the South Pole winter, particularly between Days 200-300, is expected to be quite dry (see Figure 1 in
Chamberlin 2001). Therefore, it is possible that the opacity data reported from the second half of the 1992 year is corrupted by non-ideal radiometer calibration due to the loss of proper chopper wheel phasing after Day 180 (see Section 5.3).

\subsection{Implications for Evidence of 'Dry-Air' Opacity at $225 \mathrm{GHz}$}

Previously (Chamberlin \& Bally 1995), 225-GHz opacity dependence on water vapor column, PWV, was determined by comparing concurrent measurements of both quantities. The $\tau$ versus PWV data were fitted to Equation (12)

$$
\tau=\tau_{\text {dry_air }}+B \cdot \mathrm{PWV}
$$

where $\tau_{\text {dry_air }}$ and $B$ were free parameters. PWV was obtained from a validated type of radiosonde (Chamberlin $2001), \tau_{\text {dry_air }}$ was the zero intercept, or opacity remaining at zero water vapor, and $B=\Delta \tau / \triangle \mathrm{PWV}$ (e.g. see Shimabukuro 1964).

From comparing the newly derived $\tau$ to PWV we have obtained new coefficients $\tau_{\text {dry_air }}$ and $B$ from the original 1992 skydip data set. Table 3 repeats Table 7 in Chamberlin (2001) except that a reanalysis of the 1992 data is included here.

We note that the coefficients $\tau_{\text {dry_air }}$ and $B$ resulting from the reanalysis are still in general agreement with previously reported results. However, if $\tau$ versus PWV data after Day 180 is excluded from the fit, then $\tau_{\text {dry_air }}$ is decreased slightly from 0.028 to 0.024 nepers. This exclusion may be justified since in Section 5.3 we saw that there was in the calibration system a chopper wheel phasing problem after about Day 180. If the periods inferred snow accumulation are excluded (see Section 5.4) $\tau_{\text {dry_air }}=0.026$ nepers $($ see Table 3 ).

The result that there is significant dry-air opacity at $225 \mathrm{GHz}$ is not changed by the reanalysis. 
Table 3. $\tau_{\text {dry_air }}$ and $B$ coefficients of Equation (12) derived from a linear regression fit of opacity compared to water vapor data. $R$ is the linear regression coefficient and $N$ is the number of points used in the fit. 1992 data opacity data were acquired from an NRAO 225-GHz $\tau$-meter. 1995 and 1998 opacity data were acquired with the AST/RO instrument. $v$ is the centre frequency of the double sideband heterodyne receiver used for the measurements in GHz. In both instruments, the receiver IF frequencies were centred on $1.5 \mathrm{GHz}$

NRAO documentation is ambivalent about the exact IF frequency of the $\tau$-meters. Liu (1987) states the IF centre frequency is $1.5 \mathrm{GHz}$ and spans $1 \mathrm{GHz}$. Radford et al. (2001) indicate it is $1.25 \mathrm{GHz}$ and spans $500 \mathrm{MHz}$. Examination and measurement of the NRAO $225-\mathrm{GHz} \tau$-meter at the CSO shows its IF is centred on $1.5 \mathrm{GHz}$ and spans $500 \mathrm{MHz}$. Perhaps these various reports reflect some actual differences between the four copies of the instrument

\begin{tabular}{|c|c|c|c|c|c|c|}
\hline Year & $\begin{array}{l}v \\
\mathrm{GHz}\end{array}$ & $\begin{array}{l}\tau_{\text {dry_air }} \\
\text { nepers }\end{array}$ & $\begin{array}{l}B \\
\text { nepers per } \mathrm{mmH}_{2} \mathrm{O}\end{array}$ & $R$ & $N$ & Instrument/reference \\
\hline \multirow[t]{4}{*}{1992} & 225 & 0.028 & 0.069 & 0.77 & $\sim 1100$ & $\tau$-meter/Chamberlin \& Bally (1995) \\
\hline & 225 & 0.035 & 0.081 & 0.71 & 1094 & $\tau$-meter/this reanalysis, all days \\
\hline & 225 & 0.024 & 0.084 & 0.81 & 463 & $\tau$-meter/this reanalysis, Days $\leq 180$ \\
\hline & 225 & 0.026 & 0.083 & 0.80 & 408 & $\begin{array}{l}\tau \text {-meter/this reanalysis, Days } \leq 180 \\
\text { and excluding periods with snow } \\
\text { on optics (see Section } 5.4 \text { ) }\end{array}$ \\
\hline 1995 & 229 & 0.035 & 0.10 & 0.78 & 39 & AST/RO/Chamberlin (2001) \\
\hline 1998 & 229 & 0.030 & 0.076 & 0.88 & 18 & AST/RO/Chamberlin (2001) \\
\hline
\end{tabular}

Table 4. Cumulative distribution inferred from Equation (12), Days 100-321 in 1992

\begin{tabular}{llll}
\hline Percentile & PWV & $\begin{array}{l}\tau \text { inferred } \\
\text { from Eq. (12) }\end{array}$ & $\begin{array}{l}\text { Change from Chamberlin } \\
\text { et al. (1997) }\end{array}$ \\
\hline $25 \%$ & 0.22 & 0.043 & $+11 \%$ \\
$50 \%$ & 0.34 & 0.053 & $+10 \%$ \\
$75 \%$ & 0.44 & 0.061 & $+7 \%$ \\
$99 \%$ & 1.00 & 0.108 & $+10 \%$ \\
\hline
\end{tabular}

\subsection{Inference of Cumulative Statistics for the Entire Winter Period}

As we have seen in Section 5.3, there was a problem with chopper wheel phasing after about Day 180. Thus, it is possible that the opacity data collected after Day 180 is corrupted by this problem (see Section 6.4). However, including accurate data in the cumulative statistics after Day 180 is desirable since that part of the annual cycle, on average, contains the driest portion of the year, Days 200-300.

Since we have established a relationship between radiosonde-measured PWV and $\tau$ before Day 180 , it is possible to infer the opacity after Day 180 using PWV and the derived coefficients for Equation (12).

We have made such an inference in Table 4 using $\tau_{\text {dry_air }}=0.024$ and $B=0.084$ from Table 3 . Compared to the results reported for the same period in Table 2 the increase opacity values compared to those reported in Chamberlin et al. (1997) is smaller and on the order of $10 \%$.

\subsection{Comparison to Atmospheric Transmission Model Calculation}

As has been mentioned in previous reports (Chamberlin \& Bally 1995; Chamberlin et al. 1997; Chamberlin 2001), the Grossman AT model (Grossman 1989) significantly
Table 5. Comparison to two atmospheric transmission models. Coefficients apply to zenith atmospheric transmission

\begin{tabular}{lllll}
\hline & $\begin{array}{l}\text { This reanalysis } \\
\text { Days } \leq 180\end{array}$ & AT model & ATM model & Units \\
\hline$\tau_{\text {dry_air }}$ & 0.024 & 0.005 & 0.012 & nepers \\
$B$ & 0.084 & $\begin{array}{l}0.12 \\
(\mathrm{z}-\mathrm{n} / \mathrm{typo})\end{array}$ & 0.057 & $\begin{array}{l}\text { nepers/ } \\
\mathrm{mmH}_{2} \mathrm{O}\end{array}$ \\
\hline
\end{tabular}

under-predicts the observed dry-air opacity (e.g. see Table 5). Nevertheless, the model is convenient to use and gives a rough estimate of the expected opacity. Typical of more recent models is ATM (Pardo et al. 2001) "which uses experimentally derived continuum-like terms above $300 \mathrm{GHz}$ (and extrapolated below that frequency)" to model quasi-continuum contributions to the dry- and wet-air opacities. Presented in Table 5 are the results from the ATM model for a typical South Pole wintertime atmospheric profile (1992 June 1).

\section{NRAO 225-GHz Radiometers at Other Sites}

A total of four NRAO 225-GHz heterodyne site testing radiometers were constructed (Liu 1987) for Millimetre Array site testing. Over the years, they have been used to characterise various submillimetre sites including the South Pole, Mauna Kea (Hawaii), and Chajnantor (Chile). One of the units has been in operation at the Caltech Submillimeter Observatory (CSO) since 1989. In about 1996 the CSO unit was modified with a replacement data acquisition system based on an embedded PC. The other three units are currently deployed to (1) Kitt Peak (Arizona), (2) The Combined Array for Research in Millimetrewave Astronomy (CARMA) (http://www.mmarray.org/), and (3) Chajnantor (S. Radford, private communication 2003). The unit used at the South Pole in 1992 is now probably at the CARMA site or in Chile. We note that 
systematic errors on the order of $10 \%$ or even larger may be possible with NRAO $225-\mathrm{GHz} \tau$-meters due to field modifications, gain drift, long intervals in between calibration/verification checks, and so forth. For example, during an unknown period prior to 1997 February, the unit used at the CSO reported $225-\mathrm{GHz}$ opacity values too low by $28 \%$. This deviation was established by comparisons with skydips using the CSO Leighton Telescope and its 230-GHz SIS facility receiver. Since discovery of this deviation, values reported from the CSO $\tau$-meter have had a post-correction factor included to make them agree with Leighton Telescope skydips. ${ }^{4}$

In 1994 the NRAO 225-GHz $\tau$-meter used for the 1992 South Pole opacity measurements was sent to the CSO for testing and calibration (B. Force, private communication 2002). After comparisons and internal gain adjustments to make it agree with the CSO $\tau$-meter, we believe the unit was sent on to make site survey measurements at Chajnantor in 1994 November. This unit may have been used to make measurements at Chajnantor in later years as well, but the record is currently unclear about where this specific unit travelled (S. Radford, G. Petencin, private communication 2003).

We note the following two possible problems from the 1994 CSO calibration work: the CSO $\tau$-meter was used as an opacity standard rather than skydips from the Leighton telescope; and the excess window emission may not be properly accounted for in the analysis. If there were problems in the post-1994 data produced from this $225-\mathrm{GHz}$ $\tau$-meter, then they are likely to be systematic and old data can be corrected. We note that in 2001, an NRAO 225$\mathrm{GHz} \tau$-meter used at Chajnantor was directly compared to a similar site survey instrument operating at $220-\mathrm{GHz}$ (Radford et al. 2001; figure 10). The comparisons done by Radford et al. (2001) were in relation to understanding the opacity differences between two sites in Chile being measured by two different instruments at slightly different frequencies. Although useful for their immediate purposes, for the better than $10 \%$ level of error we are interested in here, we think the comparisons did not give a definitive answer about the absolute accuracy of the $225-\mathrm{GHz}$ opacity measurements from that specific instrument.

\section{Conclusions}

This paper reexamines old 1992 skydip results from an NRAO 225-GHz heterodyne site-testing radiometer. Using an improved skydip model and considering possible sources of systematic error, we find that earlier reported opacity results were approximately $10 \%$ too low. The finding that there is significant dry-air opacity at $225 \mathrm{GHz}$ is not changed.

\footnotetext{
${ }^{4}$ In 2003 January, the original, NRAO-fabricated, whisker contacted Schottky diode mixer used in the CSO $225-\mathrm{GHz}$ heterodyne radiometer failed. In 2003 June, the mixer was replaced with a planar diode device commercially available from Virginia Diodes (model number VDI-WR3.4FM; $s / n$ 0030). To support this new mixer, a replacement, constant-current bias supply was fabricated at the CSO and installed in the CSO $\tau$-meter enclosure.
}

\section{Appendix}

The definition of $T_{\text {atm }}$ is given by (Elgered 1993)

$$
T_{\mathrm{atm}}=\frac{\int_{0}^{\infty} T \Re(T)^{-1} \alpha \exp [-\tau(s)] \mathrm{d} s}{\int_{0}^{\infty} \alpha \exp [-\tau(s)] \mathrm{d} s} .
$$

$T_{\text {atm }}$ is the effective atmospheric temperature for the single slab model, $T$ is the physical temperature of the atmosphere at height $s$ above the surface, $\Re(T)^{-1}$ converts $T$ to the equivalent radiation brightness temperature $T_{\mathrm{B}}$ (in the Rayleigh-Jeans limit $\left.T=T_{\mathrm{B}}\right), \alpha$ is the atmospheric absorbtion coefficient that applies at height $s$, and $\tau(s)$ is the total opacity from ground to the emitting layer at height $s$ (i.e. $\left.\tau(s)=\int_{0}^{s} \tau\left(s^{\prime}\right) \mathrm{d} s^{\prime}\right)$. The quantities $T_{\text {atm }}, \alpha, \Re(T)^{-1}$, and $\exp [-\tau(s)]$ are functions of the observation frequency $v$; in our case $v=225.0 \mathrm{GHz}$.

For our purpose of computing $T_{\mathrm{atm}}, \alpha$ was assumed to be due entirely to water vapor emission. To calculate $\alpha$ we included the emission from seven nearby water lines (Rosenkranz 1993)

$$
\alpha(v)=n \sum_{j=1}^{7} S_{j}(T) F\left(v, v_{j}\right)
$$

where $n$ was the number density of water molecules at height $s, v_{j}$ was the centre frequency of the water emission line with intensity $S_{j}(T)$, and $F_{j}\left(v, v_{j}\right)$ was a pressuredependent Lorentzian line-broadening function. In our calculations, the temperature $T$, pressure, and water vapor abundance at height $s$ were all determined from observations from a validated type of radiosonde (see Chamberlin 2001).

To estimate $\tau(s)$ in Equation (13) we recursively used the experimentally derived dry- and wet-air opacity coefficients $\left(\tau_{\text {dry_air }}=0.024, B=0.084\right.$, see Table 3 ) in

$$
\begin{aligned}
\tau(s)= & 0.024 \cdot\left[1-\exp \left(-2 s / s_{\mathrm{air}}\right)\right] \\
& +0.084 \cdot \mathrm{PWV} \cdot\left[1-\exp \left(-2 s / s_{\mathrm{WV}}\right)\right] .
\end{aligned}
$$

PWV was the total column of water vapor; $s_{\mathrm{Wv}}$ was the 'scale height' of water vapor, assumed to be $2 \mathrm{~km}$; $s_{\text {air }}$ was the scale height of dry air, assumed to be $8 \mathrm{~km}$. The derivation of Equation (15) assumed that $\Delta \tau(s) / \Delta s$ depended on $s$ only, however, the accuracy of it makes little difference in the computation of Equation (13) since generally $\tau(s) \ll 1$ at $225 \mathrm{GHz}$ at the South Pole.

Although this method of calculation $T_{\text {atm }}$ is somewhat elaborate, it nevertheless embodies many approximations. For example, we have only considered emission from seven nearby water vapor lines, we have neglected emission from non-water vapor species, and the functional form of $F_{j}\left(v, v_{j}\right)$ is not Lorentzian and is in fact a topic of research. Despite these approximations, we believe our method of determining is $T_{\text {atm }}$ is an improvement on assuming it is the just same as the surface temperature or just the surface temperature offset by some fixed temperature. 
It is interesting to note that computing $T_{\text {atm }}$ by just weighting the physical temperature with $n$, i.e.

$$
T_{\mathrm{atm}}^{\prime}=\frac{\int_{0}^{\infty} T n \mathrm{~d} s}{\int_{0}^{\infty} n \mathrm{~d} s}
$$

gives about the same result as using Equation (13). For example, considering wintertime Days 100-300 in 1992, the difference in the two methods gives $\left\langle T_{\mathrm{atm}}^{\prime}-\right.$ $\left.T_{\text {atm }}\right\rangle=-1.6 \pm 0.4 \mathrm{~K}$. The average difference between $T_{\mathrm{atm}}$ and the surface temperature is much larger and more random: $\left\langle T_{\text {sur }}-T_{\text {atm }}\right\rangle=-15.3 \pm 6.9 \mathrm{~K}$. $T_{\text {atm }}$ was warmer and more constant than $T_{\text {sur }}$. For the 1992 wintertime period, $\left\langle T_{\mathrm{atm}}\right\rangle=232.7 \pm 4.9 \mathrm{~K}$ whereas $\left\langle T_{\text {sur }}\right\rangle=216.4 \pm 9.3 \mathrm{~K}$.

Comparison of 220 temperature measurements from the 1992 winter season gave

$$
T_{\text {atm }}[\mathrm{K}]=0.37 T_{\text {sur }}+152
$$

with a linear correlation coefficient of 0.73 . This observed relationship was about the same as reported for the 1995 season (Ingalls 1999; Stark et al. 2001). If only $T_{\text {sur }}$ is known and some uncertainly is tolerable, $T_{\text {atm }}$ can be estimated from Equation (17).

\section{Acknowledgments}

We thank Adair Lane for providing information regarding FCRAO skydip methodology and history and Antony Stark for his comments on this report. We thank Brian Force, Simon Radford, and Gerald Petencin for providing some history about the four NRAO $225-\mathrm{GHz} \tau$-meters. We thank Juan Pardo for reading the manuscript and providing ATM model results. This work was supported by the United States Science Foundation Grants AST 9980846 and AST-0229008 to the Caltech Submillimeter Observatory.

\section{References}

Calisse P. G. 2004, PASA, 21, 257

Chamberlin, R. A., \& Bally, J. 1994, ApOpt, 33, 1095
Chamberlin, R. A., \& Bally, J. 1995, JIMW, 16, 907

Chamberlin, R. A., Lane, A. P., \& Stark, A. A. 1997, ApJ, 476, 428

Chamberlin, R. A. 2001, JGR-Atmospheres, 106 (p. 20, 101)

Chamberlin, R. A., Martin, R., Martin, C. L., \& Stark, A. A. 2003, in SPIE Proc. Ser. 4855, eds. T. G. Phillips, \& J. Zmuidzinas (Bellingham, WA: SPIE)

Davis, J. H., \& Vanden Bout, P. 1973, ApL, 15, 43

Elgered, G. 1993, in Atmospheric Remote Sensing By Microwave Radiometry, ed. M. A. Janssen (New York, NY: John Wiley \& Sons), 227

Grossman, E. 1989, AT Program, Version 1.5 (Boulder, CO: Air Head Software)

Ingalls, J. G. 1999, Ph.D. Thesis (Boston, MA: Boston Univ)

Kraus, J. D. 1986, Radio Astronomy, 2nd ed. pp. 3-32 (Powell, OH: Cygnus-Quasar)

Lane, A. P. 1978, Skydip Data Reduction Program for the FCRAO Millimeter Telescope, Five College Radio Astronomy Observatory (Amherst, MA: Univ Massachusetts)

Lane, A. P. 1982, Ph.D. Thesis (Amherst, MA: Univ Massachusetts)

Liu, Zhong-Yi. 1987, 225 GHz Atmospheric Receiver, Internal Rep. 271 (Charlottesville, VA: NRAO)

McKinnon, M. 1987, Measurement of Atmospheric Opacity Due to Water Vapor at $225 \mathrm{GHz}, \mathrm{MMA}$, Memo 40 (Charlottesville, VA: NRAO)

Meeks, M. L., \& Ruze, J. 1971, ITAP, 19, 723

Pardo, J. R., Cernicharo, J., \& Serabyn, E. 2001, ITAP, 49/12, 1683

Peterson, J. B., Radford, S. J. E., Ade, P. A. R., Chamberlin, R. A., O'Kelly, M. J., Peterson, K. M., \& Schartman, E. 2003, PASP, 115,383

Radford, S. J. E., Butler, B. J., Sakamoto, S., \& Kohno, K. 2001, Atmospheric Transparency at Chajnantor and Pampa la Bola, Memo 384 (Charlottesville, VA: NRAO)

Radford, S. J. E. 2002, in ASP Conf. Ser. 226, eds. J. Vernin, Z. Benkhaldoun, \& C. Muoz-Tun (San Francisco, CA: ASP), 148

Rosenkranz, P. W. 1993, in Atmospheric Remote Sensing by Microwave Radiometry, ed. M. A. Janssen (New York, NY: John Wiley \& Sons), 45

Rohlfs, K. 1986, Tools of Radio Astronomy (Heidelberg: Springer)

Schwerdtfeger, W. 1984, Weather and Climate of the Antarctic (New York, NY: Elsevier)

Shimabukuro, F. I. 1964, ITAP, 14, 228

Stark, A. A., Bally, J., Balm, S., Bania, T. M., Bolatto, A. D., Chamberlin, R. A., Engargiola, G., Huang, M., Ingalls, J. G., Jacobs, K., Jackson, J. M., Kooi, J. W., Lane, A. P., Lo, K. Y., Marks, R. D., Martin, C. L., Mumma, D., Ojha, R., Schieder, R., Staguhn, J., Stutzki, J., Walker, C. K., Wilson, R. W., Wright, G. A., Zhang, X., Zimmermann, P., \& Zimmermann, R. 2001, PASP, 113, 567

Ulich, B. L., \& Haas, R. W. 1976, ApJS, 30, 723 